\title{
Improved Bat Algorithm Applied to Multilevel Image Thresholding
}

\author{
Adis Alihodzic ${ }^{1}$ and Milan Tuba ${ }^{2}$ \\ ${ }^{1}$ Faculty of Mathematics, University of Sarajevo, 71000 Sarajevo, Bosnia And Herzegovina \\ ${ }^{2}$ Faculty of Computer Science, Megatrend University Belgrade, 11070 Belgrade, Serbia
}

Correspondence should be addressed to Milan Tuba; tuba@ieee.org

Received 25 April 2014; Accepted 28 June 2014; Published 3 August 2014

Academic Editor: Xin-She Yang

Copyright (C) 2014 A. Alihodzic and M. Tuba. This is an open access article distributed under the Creative Commons Attribution License, which permits unrestricted use, distribution, and reproduction in any medium, provided the original work is properly cited.

Multilevel image thresholding is a very important image processing technique that is used as a basis for image segmentation and further higher level processing. However, the required computational time for exhaustive search grows exponentially with the number of desired thresholds. Swarm intelligence metaheuristics are well known as successful and efficient optimization methods for intractable problems. In this paper, we adjusted one of the latest swarm intelligence algorithms, the bat algorithm, for the multilevel image thresholding problem. The results of testing on standard benchmark images show that the bat algorithm is comparable with other state-of-the-art algorithms. We improved standard bat algorithm, where our modifications add some elements from the differential evolution and from the artificial bee colony algorithm. Our new proposed improved bat algorithm proved to be better than five other state-of-the-art algorithms, improving quality of results in all cases and significantly improving convergence speed.

\section{Introduction}

Image segmentation is process of subdivision of an image into homogeneous and disjoint sets sharing similar properties such as intensity, color, and contours. Homogeneous sets are introduced with respect to a certain criterion of homogeneity. Image segmentation usually represents the first step in image understanding and representation and the results obtained by segmentation are used for further high-level methods such as feature extraction, semantic interpretation, image recognition, and classification of objects. In general, image segmentation simplifies the process of dividing an image into regions that are used for further specific applications. Several practical applications cover character recognition [1], detection of video changes [2], medical imaging [3, 4], automatic target recognition [5], and so forth. Over the last few decades a lot of algorithms for image segmentation, either for gray level or color images, were presented in the literature. Good review of these algorithms can be found in [6]. In general, image segmentation algorithms can be grouped into thresholding, edge-based, region-grow, and clustering.
Image thresholding is one of the most widespread segmentation techniques that performs image segmentation based on the information contained in the global gray value of the image histogram. Thresholding is called bilevel thresholding in the case that an image is separated into two classes, one including those pixels with gray levels above a specified threshold and the other including the rest. Unlike bilevel thresholding, multilevel thresholding performs subdivision of an image into several classes. In this case the pixels belonging to the same class take gray levels from the intervals defined by successive thresholds. Multiple pixels belonging to the same class are not always homogeneous and may be represented by different feature values. Selection or computing of the multilevel thresholds is crucial in image segmentation since proper segmentation depends on adequately computed thresholds.

There are many different methods for computing the thresholds for an image such as maximizing the gray level variance [7], entropy [8], similarity [9], and measure of fuzziness [10]. In general, thresholding methods can be divided 
into parametric and nonparametric methods. Using parametric methods, such as a novel image thresholding method based on Parzen window estimate [11], nonsupervised image segmentation based on multiobjective optimization [12], a multilevel thresholding approach using a hybrid optimal estimation algorithm [13], and optimal multithresholding using a hybrid optimization approach [14], may involve the solution of nonlinear equations which increases of the computational complexity. Therefore, the nonparametric methods [15] are introduced for finding the thresholds by optimizing some discriminating criteria. Among the mentioned different thresholding criteria, the entropy is the most popular optimization method. Using the entropy of the histogram, Pun was the first to introduce a new method for gray level image thresholding [8]. Later, this method was corrected and improved by Kapur et al. [16], since Kapur found some artifacts in Pun's method. Sahoo used Shanon's concept of entropy, considering two probability distributions for background and foreground objects. He has proposed a thresholding technique based on Renyi's entropy [17]. Information about the gray value of each pixel and the average value of its immediate neighborhood are obtained by twodimensional entropy which is calculated by two-dimensional histogram.

Another important group of methods based on discriminant analysis is the clustering-based methods [18]. In these methods, gray values are clustered into several classes, so that there is a similarity of gray values within the class and dissimilarity between classes. To perform the separation of classes, Otsu has developed a thresholding method for computing the optimal thresholds by maximizing the betweenclass variance using an exhaustive search [7]. It has been shown that this method gives acceptable results when the number of pixels in each class is close to each other. For bilevel image thresholding, the above-mentioned methods are effective. However, for the optimal multilevel thresholding, the existing conventional methods are being hindered by an exhaustive search when the number of thresholds is increased. To overcome this problem, powerful metaheuristics are used to search for the optimal thresholds in order to achieve a fast convergence and reduce the computational time.

Metaheuristics are optimization methods that orchestrate an interaction between local improvement procedures and higher level strategies to create a process capable of escaping from local optima and performing a robust search of a solution space $[19,20]$. Several metaheuristic algorithms derived from the behavior of biological and physical systems in the nature have been proposed as powerful methods for searching the multilevel image thresholds. Since magic algorithm that works for all problems does not exist [21], different approaches have been developed for different classes of problems such as combinatorial or continuous, with additions for constrained optimization problems [22]. Original versions of metaheuristic algorithms are often modified or hybridized in order to improve performance on some classes of problems. The most popular nature-inspired algorithms for optimization, with improvements, adjustments, and hybridizations, include particle swarm optimization (PSO) [23], differential evolution (DE) [24], firefly algorithm (FA) $[25,26]$, cuckoo search (CS) [27-29], ant colony optimization [30-33], artificial bee colony algorithm [34-38], bat algorithm (BA) [39, 40], and human seeker optimization (HSO) [41-43].

DE algorithm has been adapted for searching the optimal multilevel thresholds [44]. PSO algorithm modified by Yin to search for the thresholds can be found in [45]. Akay presented a comprehensive comparative study of the ABC and PSO algorithms for finding multilevel thresholds using Kapur's and Otsu's criteria [46]. Maitra and Chatterjee proposed an improved variant of PSO algorithm for the task of image multilevel thresholding [47]. The results showed that the $\mathrm{ABC}$ algorithm with both the between-class variance and the entropy criterion can be efficiently used in multilevel thresholding. Hammouche focused on solving the image thresholding problem by combining between-class variance criterion with metaheuristic techniques such as GA, PSO, DE, ACO, SA, and TS [48].

In this paper, we adapted the bat algorithm for multilevel image thresholding. Bat algorithm is simple to implement and produces good results. However, based on our experiments, it is powerful in intensification, but at times it may get trapped into local optima when it is applied to some difficult problems. Therefore, we propose an improved version of bat algorithm adopted to search for multilevel thresholds using Kapur and Otsu criteria. Our proposed modification merges three approaches to produce a new improved bat-inspired (IBA) algorithm according to the principle of bat algorithm, differential evolution, and some scout technique taken from the ABC algorithm. We compared our proposed algorithm with stateof-the-art algorithms from [49]. The experimental results show that the proposed IBA algorithm always gives better results compared to PSO, DE, CS, FA, and BA algorithms, considering both accuracy and, especially, convergence speed.

The remainder of the paper is organized as follows. Section 2 describes the multilevel thresholding problem and presents Kapur's and Otsu's objective functions. Section 3 and Section 4 describe the original BA and IBA algorithms adopted to search for the optimal multilevel thresholds, respectively. Section 5 shows the experimental and comparative results of applying PSO, DE, CS, FA, BA, and IBA to multilevel segmentation to standard benchmark images. Finally, our conclusions are discussed in Section 6.

\section{Multilevel Image Thresholding}

Thresholding technique performs image segmentation based on the information contained in the image histogram. If we consider a gray-scale input image $I$ as a set of pixels $A$, multilevel thresholding can be defined as a method of 
dividing the set $A$ into $n+1$ disjoint subsets $\left(A_{0}, A_{1}, \ldots, A_{n}\right)$ by some numbers $\left(\alpha_{0}, \alpha_{1}, \ldots, \alpha_{n-1}\right)$ such that

$$
\begin{aligned}
A_{0} & =\left\{x: 0 \leq f(x)<\alpha_{0}\right\}, \\
A_{1}= & \left\{x: \alpha_{0} \leq f(x)<\alpha_{1}\right\}, \\
& \vdots \\
A_{n} & =\left\{x: \alpha_{n-1} \leq f(x) \leq L-1\right\},
\end{aligned}
$$

where $x=\left(x_{1}, x_{2}\right)$ is a pixel defined by coordinates $x_{1}$ and $x_{2}$ in the Cartesian coordinate system, $f(x)$ presents a gray level value of pixel $x$, and the $f(x)$ takes values in the range $[0,255]$. The aim of multilevel thresholding is to compute the optimal threshold values $\left(\alpha_{0}, \alpha_{1}, \ldots, \alpha_{n-1}\right)$. The sets $\left(A_{0}, A_{1}, \ldots, A_{n}\right)$ may represent different regions of the object. It is clear that $A_{i} \cap A_{j}=\emptyset$, and their union presents the whole input image $I$.

Optimal threshold selection for bilevel thresholding is not computationally expensive, while for multilevel thresholding, computing more than few optimal threshold values is an expensive and time consuming operation. The optimal threshold values can be determined by optimizing some criterion functions defined from the histogram of image. In this paper, we use two popular threshold criteria: Kapur's entropy criterion and Otsu's between-class variance criterion.

2.1. Kapur's Thresholding Method. Entropy is a measure of uncertainty proposed by Shannon [50], later widely used [51]. Let $x$ be a discrete random variable taking values $x_{i}$ with probabilities $p_{i}, i=1,2, \ldots, n$, respectively. Then its entropy is defined by

$$
H(x)=-\sum_{i=1}^{n} p_{i} \ln \left(p_{i}\right)
$$

The Kapur's method [16] based on the entropy is used to perform multilevel thresholding. For this method, the threshold criteria can be formulated as follows. Assume that an image $I$ contains $n$ pixels with gray levels belonging to the set $\{0,1, \ldots, L-1\}$. Let $h(i)$ present the number of pixels at gray level $i$, and $p_{i}=h(i) / n$ is the probability of occurrences of gray level $i$ in the image $I$. The subdivision of an image into $k+1$ classes can be considered as a $k$ dimensional optimization problem for the calculation of $k$ optimal thresholds $\left(t_{0}, t_{1}, \ldots, t_{k-1}\right)$. The optimal thresholds are obtained by maximizing the objective function:

$$
f\left(t_{0}, t_{1}, \ldots, t_{k-1}\right)=\sum_{i=0}^{k} H_{i}
$$

where the entropies $H_{i}$ are defined by

$$
\begin{gathered}
H_{0}=-\sum_{i=0}^{t_{0}-1} \frac{p_{i}}{w_{0}} \ln \frac{p_{i}}{w_{0}}, \quad w_{0}=\sum_{i=0}^{t_{0}-1} p_{i}, \\
H_{1}=-\sum_{i=t_{0}}^{t_{1}-1} \frac{p_{i}}{w_{1}} \ln \frac{p_{i}}{w_{1}}, \quad w_{1}=\sum_{i=t_{0}}^{t_{1}-1} p_{i}, \\
\vdots \\
H_{k}=-\sum_{i=t_{k-1}} \frac{p_{i}}{w_{k}} \ln \frac{p_{i}}{w_{k}}, \quad w_{k}=\sum_{i=t_{k-1}}^{L-1} p_{i} .
\end{gathered}
$$

2.2. Otsu's Thresholding Method. Otsu's method [7] based on the maximization of the between-class variance is one of the most popular methods proposed for image thresholding. The algorithm for this method can be described as follows. Assume that an image $I$ can be represented by $L$ gray levels. The probabilities of pixels at level $i$ are denoted by $p_{i}$ so $p_{i} \geq 0$ and $p_{0}+p_{1}+\cdots+p_{L-1}=1$. Cumulative probabilities for classes $A_{i}, i=0,1, \ldots, k$, can be defined as

$$
w_{0}=\sum_{i=0}^{t_{0}-1} p_{i}, \quad w_{1}=\sum_{i=t_{0}}^{t_{1}-1} p_{i}, \ldots, w_{k}=\sum_{i=t_{k-1}}^{L-1} p_{i},
$$

where $t_{j}$ are the thresholds separating these classes. For $k+1$ classes $A_{i},(i=0,1, \ldots, k)$, the goal is to maximize the objective function:

$$
f\left(t_{0}, t_{1}, \ldots, t_{k-1}\right)=\sum_{i=0}^{k} \sigma_{i}
$$

where the sigma functions are defined by

$$
\begin{aligned}
\sigma_{0} & =w_{0}\left(\sum_{i=0}^{t_{0}-1} \frac{i p_{i}}{w_{0}}-\sum_{i=0}^{L-1} i p_{i}\right)^{2}, \\
\sigma_{1} & =w_{1}\left(\sum_{i=t_{0}}^{t_{1}-1} \frac{i p_{i}}{w_{1}}-\sum_{i=0}^{L-1} i p_{i}\right)^{2}, \\
& \vdots \\
\sigma_{k} & =w_{k}\left(\sum_{i=t_{k-1}}^{L-1} \frac{i p_{i}}{w_{k}}-\sum_{i=0}^{L-1} i p_{i}\right)^{2} .
\end{aligned}
$$

\section{Bat Algorithm Adapted for Multilevel Image Thresholding}

Bat algorithm is a recent metaheuristic introduced by Yang [39], based on so-called echolocation of the bats. In this algorithm, bats detect prey and avoid the obstacles by using the echolocation. Bat algorithm was successfully applied to a number of very different problems like large-scale optimization problems [52], global engineering optimization 
[53], fuzzy clustering [54], parameter estimation in dynamic biological systems [55], multiobjective optimization [56], image matching [57], economic load and emission dispatch problems [58], data mining [59], scheduling problems [60], neural networks [61], and phishing website detection [62].

In the bat algorithm, bats navigate by using time delay from emission to the reflection. The pulse rate can be simply determined in the range from 0 to 1 , where 0 means that there is no emission and 1 means that the bat's emitting is at maximum. Apart from the control parameters, such as the population size and maximum iteration number which are common control parameters for all nature inspired algorithms, the BA has few important parameters such as frequency tuning parameter similar to the key feature used in the PSO and HS, parameter for automatically zooming into a region where the promising solutions have been found, and the control parameter for automatically switching from exploration to exploitation. This gives advantage to the BA over other metaheuristic algorithms in the literature.

In order to implement the bat algorithm, the following three idealized rules are used [39]:

(i) all bats use echolocation to sense distance, and they also "know" the surroundings in some magical way;

(ii) bats fly randomly with velocity $v_{i}$ at position $x_{i}$ with a fixed frequency $f_{\min }$, varying wavelength $\lambda$, and loudness $A_{0}$ to search for prey. They can automatically adjust the wavelength of their emitted pulses and adjust the rate of pulse emission $r$ from $[0,1]$, depending on the proximity of their target;

(iii) although the loudness can vary in many ways, it is assumed that the loudness varies from a positive large value $A_{0}$ to a minimum constant value $A_{\text {min }}$.

The proposed bat algorithm tries to select $k$ threshold values which maximize the fitness functions which are described by (3) and (6), respectively. The details of the developed BA approach for multilevel image thresholding are given as follows.

Step 1 (generate initial population of solutions). The bat algorithm generates a randomly distributed initial population of $N$ solutions (bats) $(i=1,2, \ldots, N)$, where each solution has $k$ dimensions. All solutions can be presented by matrix $X$ :

$$
X=\left[\begin{array}{ccccc}
x_{1,1} & x_{1,2} & x_{1,3} & \cdots & x_{1, k} \\
x_{2,1} & x_{2,2} & x_{2,3} & \cdots & x_{2, k} \\
& & & & \vdots \\
x_{N, 1} & x_{N, 2} & x_{N, 3} & \cdots & x_{N, k}
\end{array}\right],
$$

where $x_{i, j}$ is the $j$ th component value that is restricted to $\{0, \ldots, L-1\}$ and $x_{i, j}<x_{i, j+1}$ for all $j$. The fitness values for all solutions are evaluated and variable cycle is set to one. The bat algorithm detects the most successful solution as $x_{\text {best }}$ before starting iterative search process.

Step 2 (calculation of new solutions). Calculation of a new solution $x_{i}^{t}$ is performed by moving virtual bats $x_{i}^{t-1}$ according to equation

$$
x_{i}^{t}=x_{i}^{t-1}+v_{i}^{t},
$$

where $v_{i}^{t}$ denotes the bat velocity of movement, and it is calculated by formula

$$
v_{i}^{t}=v_{i}^{t-1}+\left(x_{i}^{t}-x_{\text {best }}\right) * f_{i} .
$$

In (10), $f_{i}$ denotes the frequency and $x_{\text {best }}$ denotes the current global best solution. The frequency $f_{i}$ can be calculated as

$$
f_{i}^{t}=f_{\min }+\left(f_{\max }-f_{\min }\right) * \beta,
$$

where $\beta$ is a random vector generated by a uniform distribution belonging to the closed interval $[0,1]$. For min and $\max$ frequency, the recommended values $f_{\min }=0$ and $f_{\max }=2$ are used. In this computation step, the bat algorithm controls the boundary conditions of the calculated new solution $x_{i}^{t}$. In the case that the value of a variable overflows the allowed search space limits, then the value of the related variable is updated with the value of the closer limit value to the related variable.

Step 3 (improving the current best solution). For each solution $x_{i}^{t}$ apply the next operator which is defined by

$$
x_{\text {new }}= \begin{cases}x_{\text {best }}+\epsilon A_{t}, & \text { if } \operatorname{rand}_{1}>r_{i}^{t}, \\ x_{i}^{t}, & \text { otherwise, }\end{cases}
$$

where rand $_{1}$ is a uniform random number in range $[0,1], \epsilon$ is a scaling factor drawn from uniform distribution in the range $[-1,1], A_{t}=\left\langle A_{i}^{t}\right\rangle$ is the average loudness of all bats at the computation step $t$, and $r_{i}^{t}$ is the pulse rate function. The pulse rate function is defined by

$$
r_{i}^{t}=r_{i}^{0}\left(1-e^{-\beta t}\right)
$$

where $\beta$ is a constant and $r_{i}^{0}$ are initial pulse rates in the range $[0,1]$. It can be seen from (12) that this function controls the intensive local search depending on the value of uniform variable rand $_{1}$ and the rate $r_{i}^{t}$. Also, at this step, the BA controls the boundary conditions at each iteration.

Step 4 (acceptation of a new solution by flying randomly). In this step, the solution $x_{\text {new }}$ obtained in Step 3 is accepted as a new solution and $f\left(x_{\text {new }}\right)$ as a new objective function value by using

$$
\left(x_{i}^{t}, \text { fit }\left(x_{i}^{t}\right)\right)=\left\{\begin{array}{l}
\left(x_{\text {new }}, f\left(x_{\text {new }}^{t}\right)\right), \\
\quad \text { if }\left(\text { rand }_{2}<A_{i}^{t} \text { and } f\left(x_{\text {new }}^{t}\right)>f\left(x_{i}^{t-1}\right)\right), \\
\left(x_{i}^{t-1}, f\left(x_{i}^{t-1}\right)\right), \text { otherwise, }
\end{array}\right.
$$

where rand $_{2}$ is a uniform random number in range $[0,1]$ and $A_{i}^{t}$ is the loudness function defined by

$$
A_{i}^{t}=\alpha A_{i}^{t-1}
$$


where $\alpha$ is a constant and plays a similar role as the cooling factor of a cooling schedule in the simulated annealing. Therefore, if the solution $x_{\text {new }}$ has the higher objective function value compared to the old solution $x_{i}^{t-1}$ and the loudness $A_{i}^{t}$ is more than rand $_{2}$, then the new solution is accepted, the old fitness value is updated, and functions defined by (13) and (15) are updated, too. Otherwise, the new solution $x_{\text {new }}$ is abandoned, and the old best solution is kept.

Step 5 (memorize the best current solution). Record the best solution so far $\left(x_{\text {best }}\right)$, that is, the solution with the highest objective function value.

Step 6 (check the stopping criteria). If the termination criterion is met or the variable cycle is equal to the maximum number of iterations, then the algorithm is finished. Otherwise, increase the variable cycle by one and go to Step 2.

\section{Our Proposed Improved Bat Algorithm: IBA}

As described in the previous section, we selected the BA for multilevel image thresholding. BA is simple to implement and it produces good results when the number of thresholds is small. However, based on our experiments, the BA algorithm often fails when the number of thresholds is larger, especially for the Kapur's objective function. Therefore, an adjustment of the bat algorithm was required. In this paper, the improved hybridized bat algorithm (IBA) is proposed to overcome the mentioned drawback of the pure bat algorithm. It combines two different solution search equations of the bat algorithm and DE algorithm [24]. The IBA algorithm includes differential operators mutation and crossover from DE algorithm, with the aim of speeding up convergence and to achieve a good balance between intensification and diversification. Mutation and crossover operators are used to improve the original BA generation of a new solution for each bat so that the IBA can more efficiently explore and exploit the new search space and avoid being trapped into local optima.

In the pure BA, exploration and exploitation are controlled by pulse rate function (13). Analyzing this function we noticed that the algorithm loses exploration capability as iterations progress. The form of this function makes switching from the exploration to exploitation and vice versa possible. In this way, the exploration capability of BA can be modified by inserting differential operators for crossover and mutation [24] instead of (12) and for the exploitation capability (12) continues to be used for a good intensification. Therefore, a good balance is established between intensification and diversification.

Although the above modification can improve many solutions, some solutions will still remain stuck in some local optimum. In order to fix this lack of the former modification, we introduced the second modification which is inspired by launch of the scouts in the scout phase of the $\mathrm{ABC}$ algorithm. When some solution gets trapped in a local optimum after a certain number of iterations, it will eventually exceed the predetermined number of allowed trials called "limit." When a solution exceeds the "limit" trials unchanged, it is redirected to search new space by using the random walk.
In the proposed IBA algorithm, bats form a population of threshold values. The threshold values produced by the bat $i$ are noted as $x_{i}=\left(x_{i, 1}, x_{i, 2}, \ldots, x_{i, k}\right), i=1, \ldots, N$. All bats perform searching in the solution search space with the aim to optimize the objective functions described by (3) or (6). The details of the proposed IBA approach for multilevel thresholding are given as follows.

Step 1 (generate the initial population of solutions). The IBA begins by randomly generating population with $k$ dimensions as in the case of the proposed BA approach for multilevel thresholding. Each threshold value $x_{i, j}(i=1, \ldots, n ; j=$ $1, \ldots, k)$ of the matrix $X$ generated by the bat $i$ is restricted to set $\{0,1, \ldots, L-1\}$ and for all $j$ holds $x_{i, j}<x_{i, j+1}$. Also, at this step initialization is done for the parameter limit which presents the number of allowed attempts to improve a bat, the initial loudness $A_{i}$ and pulse rate $r_{i}^{0}$, as well as the initial values of the parameters in the $\mathrm{DE}$ algorithm such as the differential weight $F$ and crossover probability $C_{r}$. After generation of the initial population, the fitness value for each solution $x_{i}$ is evaluated. Then the IBA algorithm detects the most successful solution as $x_{\text {best }}$, before starting iterative search process. After that it sets the variable cycle to one.

Step 2 (calculate the new population). Calculation of a new threshold $x_{i}^{t}$ is performed by moving virtual bats $x_{i}^{t-1}$ according to (9). The velocity $v_{i}^{t}$ and frequency $f_{i}$ are calculated by (10) and (11), respectively. At this computation step, the IBA controls the boundary conditions of the calculated new solution $x_{i}^{t}$. In the case that the value of the $x_{i}^{t}$ is less than 0 or is more than $L-1$, then the value of the $x_{i}^{t}$ is updated with the value of the closer limit value to the variable $x_{i}^{t}$.

Step 3 (improving the current best solution by differential operators). For each solution $x_{i}^{t}$ apply the next operator which is defined by

$$
x_{\text {new }}= \begin{cases}x_{\text {dif }}^{t}, & \text { if } \text { rand }_{1}>r_{i}^{t}, \\ x_{\text {loc }}^{t}, & \text { otherwise }\end{cases}
$$

where rand $_{1}$ is randomization term in the range $[0,1], r_{i}^{t}$ is the pulse rate function defined by (13), $x_{\text {dif }}^{t}$ is the differential operator for mutation and crossover, and $x_{\text {loc }}^{t}$ is the operator based on the local search in the BA. The differential mutation and crossover operations are performed by

$$
x_{\mathrm{dif}, j}^{t}= \begin{cases}x_{c, j}^{t}+F\left(x_{a, j}^{t}-x_{b, j}^{t}\right), & \text { if }\left(\text { rand }_{2}<C_{r} \text { or } j=j_{r}\right), \\ x_{i, j}^{t}, & \text { otherwise }\end{cases}
$$

where $x_{a}, x_{b}$, and $x_{c}$ are three randomly chosen different vectors in the range $[0, N-1]$ at the cycle $t, F$ is the differential weight that scales the rate of modification, $C_{r}$ is the crossover probability in the interval $[0,1], j_{r}$ is randomly selected in the range $[0, k]$, and $\mathrm{rand}_{2}$ is a uniform variable in the range $[0,1]$. Inside the implementation of the differential operator $x_{\mathrm{dif}}$, the boundary conditions for all $j(j=1, \ldots, k)$ are controlled. As an important improvement of the proposed method, the binomial "DE/rand/1/bin" scheme is used in 
order to increase the diversity of the bats and achieve both the precision and search efficiency. The local search is performed by

$$
x_{\mathrm{loc}, j}^{t}= \begin{cases}x_{l \mathrm{best}, j}^{t}, & \text { if }\left(f\left(x_{l \mathrm{best}, j}^{t}\right)>f\left(x_{i, j}^{t}\right)\right), \\ x_{i, j}^{t}, & \text { otherwise, }\end{cases}
$$

where $x_{l \mathrm{best}, j}^{t}$ is defined by

$$
x_{\text {lbest }, j}^{t}=x_{\text {best }, j}^{t-1}+\epsilon A_{i, j}^{t-1} .
$$

As in the ordinary BA, parameters $\epsilon$ and $A_{i, j}$ denote the scaling factor and the loudness function, respectively. Also, inside the local search operator $x_{\text {loc }}$, the boundary conditions for all $j(j=1, \ldots, k)$ are checked. In our proposed approach, we found that it is beneficial to replace (13) by $r_{i}^{t}=r_{i}^{0}\left(1-\beta^{t}\right)$. It will be shown in experimental study that the best results are obtained for initial pulse rates $r_{i}^{0}=0.5$, initial loudness $A_{0}=0.95$, and $\beta=0.9$.

Step 4 (acceptation of a new solution by flying randomly). In this step, the solution $x_{\text {new }}$ obtained in Step 3 is accepted as a new solution and $f\left(x_{\text {new }}\right)$ as a new objective function value by using

$$
\left(x_{i}^{t}, \text { fit }\left(x_{i}^{t}\right)\right)=\left\{\begin{array}{l}
\left(x_{\text {new }}^{t}, f\left(x_{\text {new }}^{t}\right)\right), \\
\quad \text { if }\left(\text { rand }_{3}<A_{i}^{t} \text { and } f\left(x_{\text {new }}^{t}\right)>f\left(x_{i}^{t-1}\right)\right), \\
\left(x_{i}^{t-1}, f\left(x_{i}^{t-1}\right)\right) \quad \operatorname{tr}_{i}=\operatorname{tr}_{i}+1, \\
\text { otherwise, }
\end{array}\right.
$$

where rand $_{3}$ is a random number in the range $[0,1], t r_{i}$ is a vector recording the number of attempts through which solution $x_{i}^{t}$ could not be improved at cycle $t$, and $A_{i}^{t}$ is defined by (15). In the above equation, if the solution $x_{i}^{t-1}$ cannot be improved, then the new solution $x_{\text {new }}$ is abandoned and the $i$ th element of the trial vector $t r$ is increased by one. Also, after certain number of cycles determined by the variable limit, if the solution $x_{i}^{t}$ cannot be further improved, it is abandoned and replaced by randomly generated solution. In this case, the $i$ th element of the trial vector is set to 0 . This modification can improve the exploration process and it will help to avoid trapping into some local optima. Also, it will improve the solution quality and speed convergence.

Step 5 (memorize the best current solution). Record the best solution so far $\left(x_{\text {best }}\right)$, that is, the solution with the highest objective function value.

Step 6 (check the stopping criteria). If the termination criterion is met or the variable cycle is equal to the maximum number of iterations, then the algorithm is finished. Otherwise, increase the variable cycle by one and go to Step 2 .

\section{Experimental Results}

The multilevel image thresholding problem deals with finding optimal thresholds within the range $[0, L-1]$ that maximize the functions defined by (3) and (6). The dimension of the optimization problem is the number of thresholds $k$, and the search space is $[0, L-1]^{k}$. In this study our proposed IBA algorithm was compared against four other standard population based metaheuristic techniques: PSO, DE, CS, and FA from [49] and pure BA.

The experiments were conducted on 6 standard images, the same as used in [49], in order to make comparison of the obtained results simpler. Images used in this paper, namely, Barbara, Living room, Boats, Goldhill, and Lake, are of size $(512 \times 512)$ and Aerial has size $(256 \times 256)$. Barbara and Boats images are available at http://decsai.ugr .es/ javier/denoise/test_images/. The Living room and Lake images were chosen from http://www.imageprocessingplace .com/root_files_V3/image_databases.htm. The Goldhill image can be found at https://ece.uwaterloo.ca/ z70wang/ research/quality_index/demo.html. The Aerial image was taken from the University of Southern California Signal and Image Processing Institute's image database at http://sipi.usc .edu/database/database.php?volume $=$ misc. These original images and their gray level histograms are depicted in Figures 1 and 2 , respectively.

For the Kapur's and Otsu's thresholding methods, the exhaustive search method was conducted first to derive the optimal solutions, the corresponding optimal objective function values, and the processing time for comparison with the results generated by the PSO, DE, CS, FA, BA, and IBA algorithms. These results generated by the exhaustive search for Kapur's and Otsu's criterion are presented in Tables 1 and 2, respectively. It is obvious that computational times increase exponentially and for more than 5 thresholds become unacceptable. We did not implement optimal use of multicore processor, but improvements would not be significant.

The number of thresholds $k$ explored in the experiments were 2, 3, 4, and 5. Since metaheuristic algorithms have stochastic characteristics, each experiment was repeated 50 times for each image and for each $k$ value. Each run of an algorithm was terminated when the fitness value of the best solution $f\left(x_{\text {best }}\right)$ reached the known optimal value (from the exhaustive search) of the objective function $f_{\text {opt }}$, that is, $\left|f\left(x_{\text {best }}\right)-f_{\text {opt }}\right|<\epsilon$, where $\epsilon=10^{-9}$ was a tolerance for the accuracy of the measurement. Hence, the stopping condition for all algorithms was the value of the fitness, unless optimum could not be reached within 2000 iterations.

The proposed IBA method has been implemented in C\# programming language, as the rest of the algorithms. Results for CS and FA are from [49]. All tests were done on an Intel Core i7-3770K @3.5 GHz with 16 GB of RAM running under the Windows 8 x64 operating system. The PSO and $\mathrm{DE}$ algorithms have been implemented in their basic versions, while the BA and IBA have been implemented as it was described in the previous two sections.

5.1. Parameters Setup. To compare the proposed IBA algorithm with PSO, DE, CS, FA [49], and BA algorithms, the objective function evaluation was computed $N \times G$ times, where $N$ is the population size and $G$ is the maximum number of generations (unless optimum was reached earlier). 


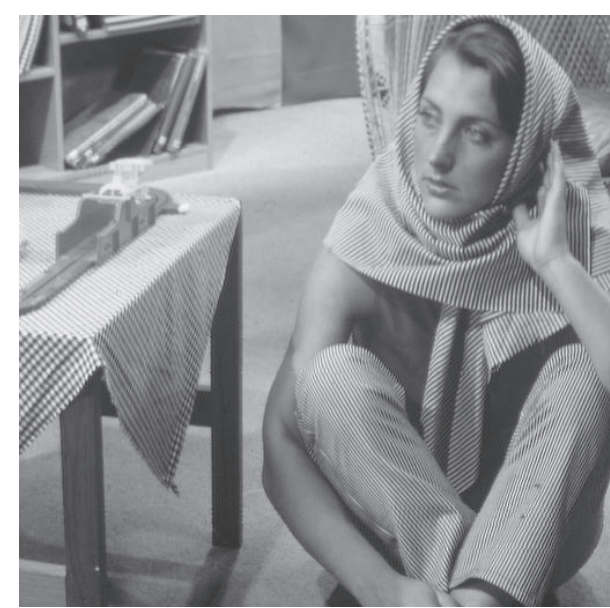

(a)

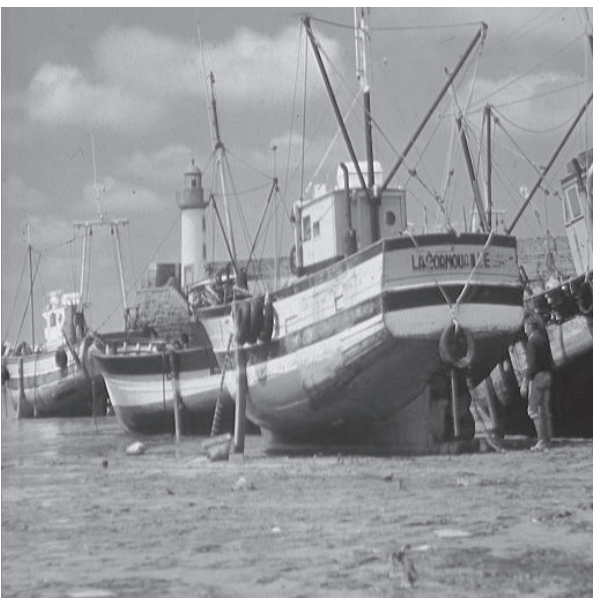

(c)

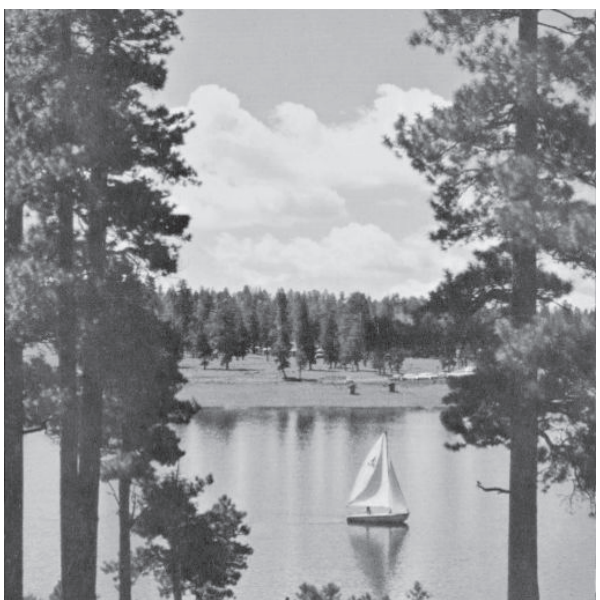

(e)

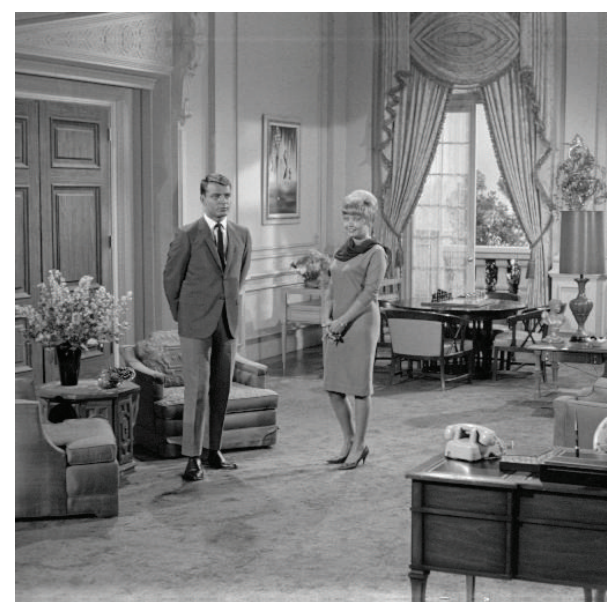

(b)

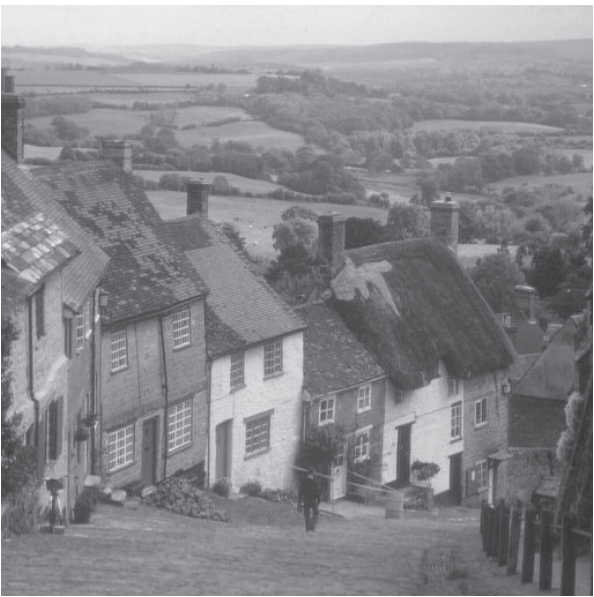

(d)

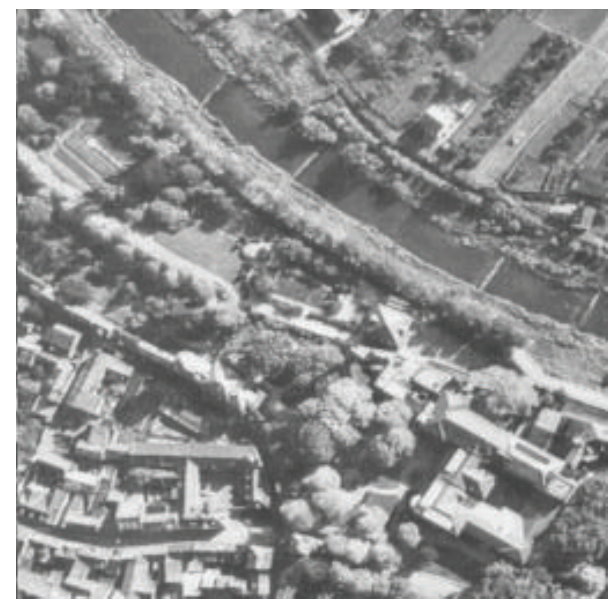

(f)

Figure 1: Test images: (a) Barbara, (b) Living room, (c) Boats, (d) Goldhill, (e) Lake, and (f) Aerial.

The population size in all algorithms was set to $N=40$ and the number of generation is set to $G=2000$ for all algorithms, as in [49]. Besides these common control parameters, each of mentioned algorithms has additional control parameters that directly improve their performance.
For both the proposed IBA and pure BA algorithms, the additional control parameters $f_{\min }$ and $f_{\max }$ were set to 0 and 2.0, respectively. The initial values for parameters $r_{i}^{0}$ and loudness $A_{i}$ were set to 0.5 and 0.99 , respectively. The constant $\beta$ was set to 0.9 . Instead of the average loudness $\left\langle A_{i}^{t}\right\rangle$ 


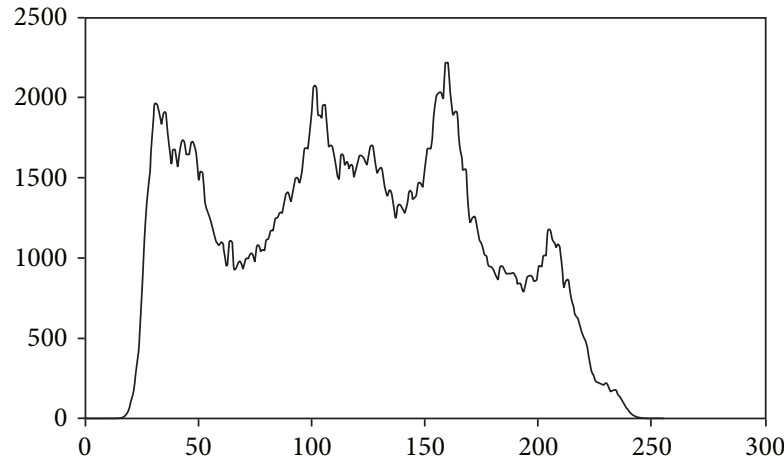

(a)

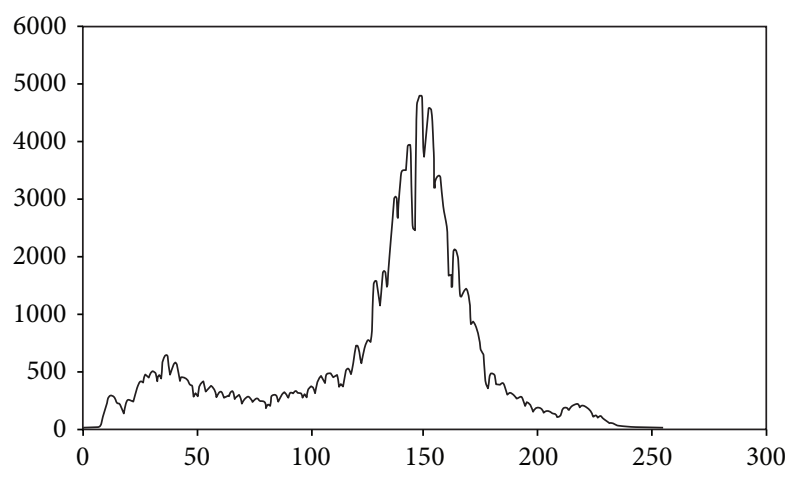

(c)

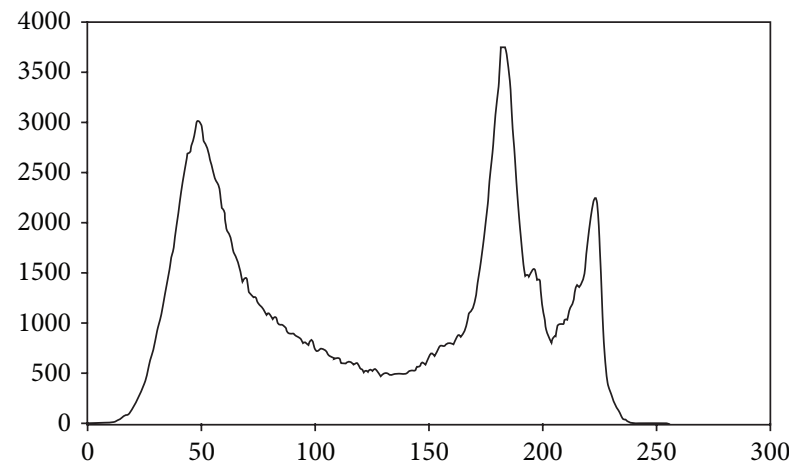

(e)

Figure 2: Gray-level histogram of test images: (a) Barbara, (b) Living room, (c) Boats, (d) Goldhill, (e) Lake, and (f) Aerial.

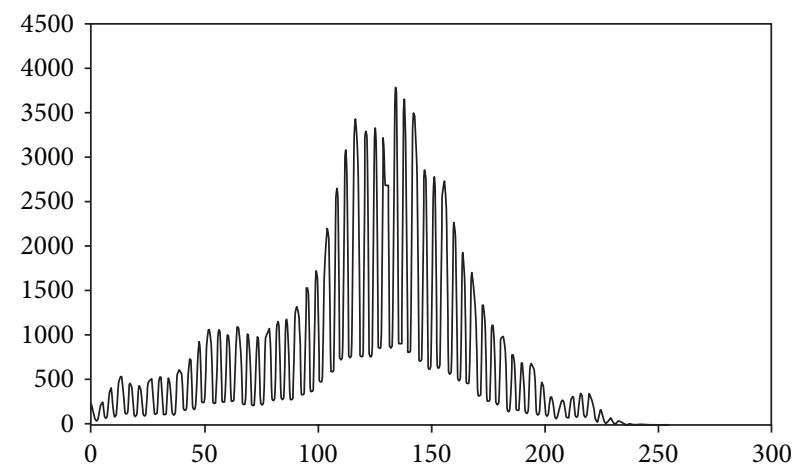

(b)

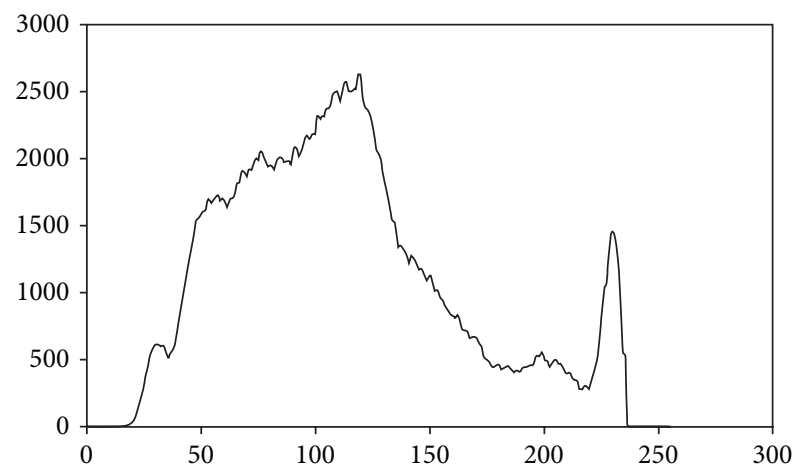

(d)

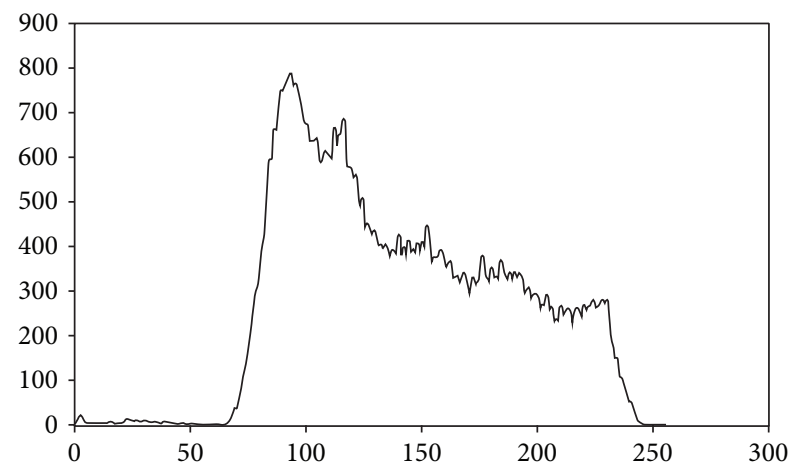

(f) of all bats, we found that the value 1.66 was acceptable for all images. In the proposed IBA algorithm, control parameters introduced from DE algorithm, such as differential weight $F$ and crossover probability $C_{r}$, were set to 0.75 and 0.95 , respectively. Also, in the IBA method, the parameter limit was set to 150 .

5.2. Quality and Computational Analysis of the Results. The mean and standard deviations for 50 runs for six tested metaheuristic algorithms have been calculated and are presented in Table 3 for the experiments based on Kapur's entropy and in Table 4 for the experiments based on Otsu's objective function. These mean values can be compared to the optimal values of the corresponding objective functions found by an exhaustive search from Tables 1 and 2.

The first conclusion that can be drown from the results in Tables 3 and 4 is that the cases when the number of desired thresholds is 2 or 3 are too easy and are not interesting for nondeterministic metaheuristics. Almost all algorithms in almost all cases reached optimal results (PSO and DE had few misses). We included these results in the tables for comparison with results in [49], but we will not discuss them further. All the remaining discussion is only about cases when the number of desired thresholds is 4 or 5 .

From Tables 3, 4, 5, and 6 many details can be seen. We will here, in three additional tables, synthetize the most 
TABLE 1: Thresholds, objective function values, and time processing provided by the exhaustive search for Kapur's method.

\begin{tabular}{|c|c|c|c|c|}
\hline Images & K & $\begin{array}{c}\text { Threshold } \\
\text { values }\end{array}$ & $\begin{array}{l}\text { Objective } \\
\text { function }\end{array}$ & Time (ms) \\
\hline \multirow{4}{*}{ Barbara } & 2 & 96,168 & 12.668336540 & 25 \\
\hline & 3 & $76,127,178$ & 15.747087798 & 341 \\
\hline & 4 & $\begin{array}{c}60,99,141 \\
185\end{array}$ & 18.556786861 & 11103 \\
\hline & 5 & $\begin{array}{c}58,95,133 \\
172,210\end{array}$ & 21.245645310 & 666869 \\
\hline \multirow{4}{*}{ Living room } & 2 & 94,175 & 12.405985592 & 31 \\
\hline & 3 & $47,103,175$ & 15.552622213 & 339 \\
\hline & 4 & $\begin{array}{c}47,98,149 \\
197\end{array}$ & 18.471055578 & 12612 \\
\hline & 5 & $\begin{array}{c}42,85,124 \\
162,197\end{array}$ & 21.150302316 & 478114 \\
\hline \multirow{4}{*}{ Boats } & 2 & 107,176 & 12.574798244 & 25 \\
\hline & 3 & $64,119,176$ & 15.820902860 & 342 \\
\hline & 4 & $\begin{array}{c}48,88,128 \\
181\end{array}$ & 18.655733570 & 11461 \\
\hline & 5 & $\begin{array}{c}48,88,128 \\
174,202\end{array}$ & 21.401608305 & 469862 \\
\hline \multirow{4}{*}{ Goldhill } & 2 & 90,157 & 12.546393623 & 24 \\
\hline & 3 & $78,131,177$ & 15.607747002 & 329 \\
\hline & 4 & $\begin{array}{c}65,105,147 \\
189\end{array}$ & 18.414213765 & 11958 \\
\hline & 5 & $\begin{array}{c}59,95,131 \\
165,199\end{array}$ & 21.099138996 & 399458 \\
\hline \multirow{4}{*}{ Lake } & 2 & 91,163 & 12.520359742 & 24 \\
\hline & 3 & $72,119,169$ & 15.566286745 & 336 \\
\hline & 4 & $\begin{array}{c}70,111,155 \\
194\end{array}$ & 18.365636309 & 12658 \\
\hline & 5 & $\begin{array}{c}64,99,133 \\
167,199\end{array}$ & 21.024982760 & 410753 \\
\hline \multirow{4}{*}{ Aerial } & 2 & 68,159 & 12.538208248 & 29 \\
\hline & 3 & $68,130,186$ & 15.751881495 & 347 \\
\hline & 4 & $\begin{array}{c}68,117,159 \\
200\end{array}$ & 18.615899102 & 11390 \\
\hline & 5 & $\begin{array}{c}68,108,141 \\
174,207\end{array}$ & 21.210455499 & 599570 \\
\hline
\end{tabular}

important conclusions concerning the quality of the results and the convergence speed.

Table 7, computed from Tables 3 and 4, shows for each tested algorithm in what percentage of cases it achieved the best result, considering all tested images and both optimization criteria. From Table 7, we can see that PSO and DE were very inferior compared to other tested algorithms. The results for the CS and FA [49] algorithms are quite acceptable, where FA had slightly better results.

For the BA we can notice that it gives rather poor results for the Kapur's method, while it gives rather good results for the Otsu's method. When the Kapur's criterion is used, the BA gets trapped in local optima, so it consumes the maximum number of iterations without switching to another subspace
TABLE 2: Thresholds, objective function values, and time processing provided by the exhaustive search for Otsu's method.

\begin{tabular}{|c|c|c|c|c|}
\hline Images & K & $\begin{array}{c}\text { Threshold } \\
\text { values }\end{array}$ & $\begin{array}{l}\text { Objective } \\
\text { function }\end{array}$ & Time (ms) \\
\hline \multirow{4}{*}{ Barbara } & 2 & 82,147 & 2608.610778507 & 39 \\
\hline & 3 & $75,127,176$ & 2785.163280467 & 89 \\
\hline & 4 & $66,106,142,182$ & 2856.262131671 & 3014 \\
\hline & 5 & $\begin{array}{c}57,88,118,148 \\
184\end{array}$ & 2890.976609405 & 100079 \\
\hline \multirow{4}{*}{ Living room } & 2 & 87,145 & 1627.909172752 & 39 \\
\hline & 3 & $76,123,163$ & 1760.103018395 & 88 \\
\hline & 4 & $56,97,132,168$ & 1828.864376614 & 2945 \\
\hline & 5 & $\begin{array}{c}49,88,120,146 \\
178\end{array}$ & 1871.990616316 & 130397 \\
\hline \multirow{4}{*}{ Boats } & 2 & 93,155 & 1863.346730649 & 38 \\
\hline & 3 & $73,126,167$ & 1994.536306242 & 89 \\
\hline & 4 & $65,114,147,179$ & 2059.866280428 & 2931 \\
\hline & 5 & $\begin{array}{c}51,90,126,152 \\
183\end{array}$ & 2092.775965336 & 75879 \\
\hline \multirow{4}{*}{ Goldhill } & 2 & 94,161 & 2069.510202452 & 38 \\
\hline & 3 & $83,126,179$ & 2220.372641501 & 88 \\
\hline & 4 & $69,102,138,186$ & 2295.380469158 & 2775 \\
\hline & 5 & $\begin{array}{c}63,91,117,147 \\
191\end{array}$ & 2331.156597921 & 74674 \\
\hline \multirow{4}{*}{ Lake } & 2 & 85,154 & 3974.738214185 & 39 \\
\hline & 3 & $78,140,194$ & 4112.631097687 & 89 \\
\hline & 4 & $67,110,158,198$ & 4180.886161109 & 2613 \\
\hline & 5 & $\begin{array}{c}57,88,127,166 \\
200\end{array}$ & 4216.943583790 & 73019 \\
\hline \multirow{4}{*}{ Aerial } & 2 & 125,178 & 1808.171050536 & 46 \\
\hline & 3 & $109,147,190$ & 1905.410606582 & 103 \\
\hline & 4 & $104,134,167,202$ & 1957.017965982 & 2670 \\
\hline & 5 & $\begin{array}{c}99,123,148,175 \\
205\end{array}$ & 1980.656737348 & 99880 \\
\hline
\end{tabular}

which is more promising. That explains why it needed some modifications to be introduced to help it leave the local optimum space and continue to search new spaces.

Our proposed improved IBA algorithm, by taking some features of the $\mathrm{DE}$ and $\mathrm{ABC}$ algorithms, obtained the best results compared to the rest of algorithms. It actually achieved the best result for both mean value and variance, for all tested cases.

Tables 5 and 6 report the mean number of iterations and the average CPU time taken by each algorithm to satisfy the stopping condition for Kapur's and Otsu's criteria, respectively. Most significant conclusions concerning the convergence speed of the tested algorithms are shown in Tables 8 and 9.

In Table 8 (for Kapur's criterion) in each column labeled by Thrs. $k(k=2,3,4,5)$ we calculated for each of the tested algorithms: PSO, DE, CS, FA, BA, and IBA, the sum of mean number of required iterations for each test image. We can observe that in the case of the FA and especially the IBA 


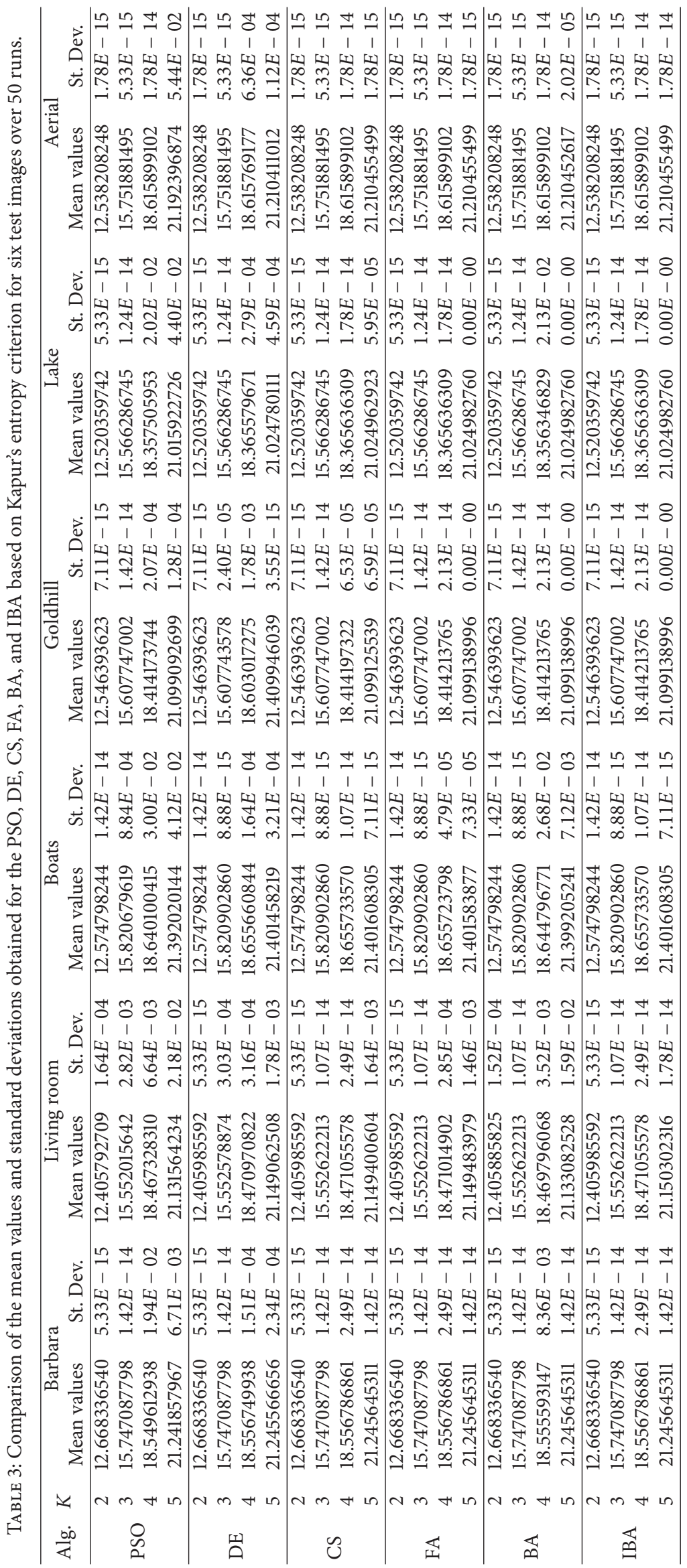


年 营

T.

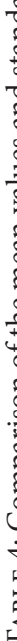
:

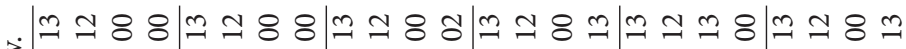

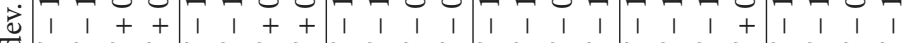

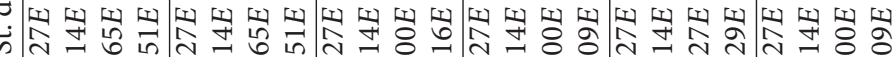
A

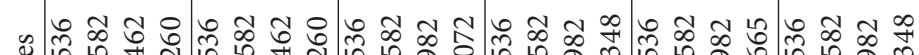

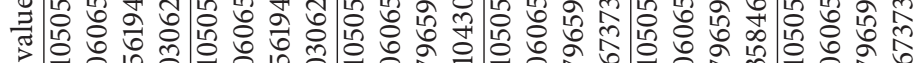
כ 국 艺

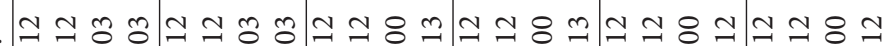

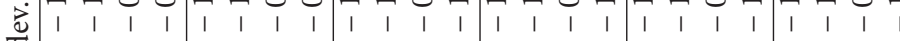

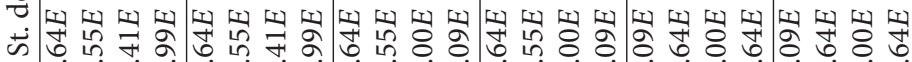

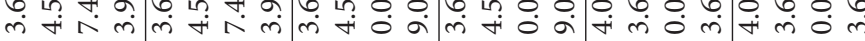

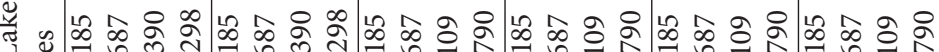

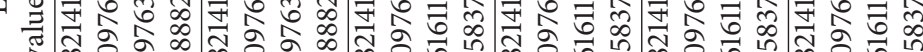

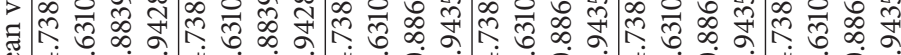

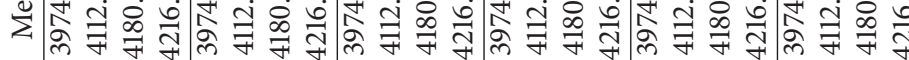

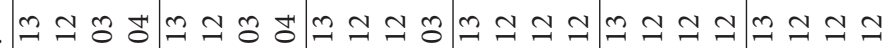

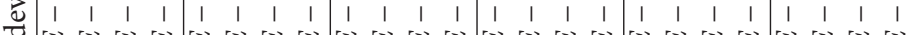

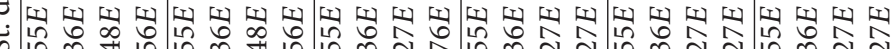
= 宾

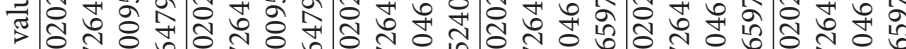

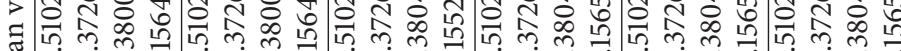
范芯 . ฉำช

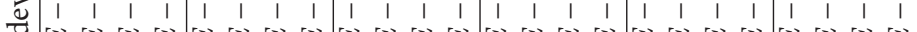

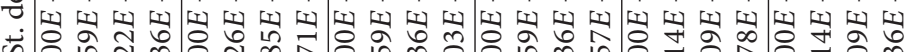
萑

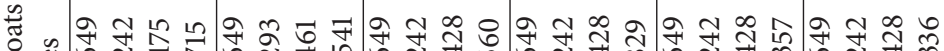

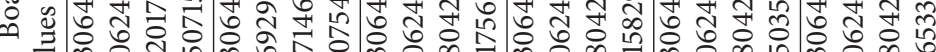

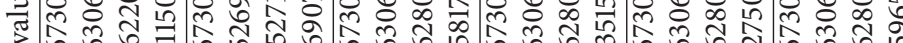

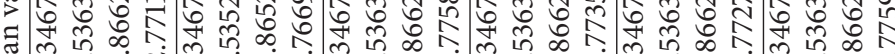

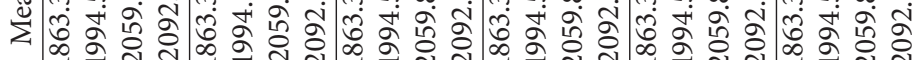
.

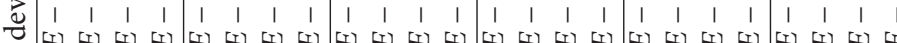

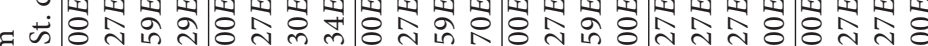
פ

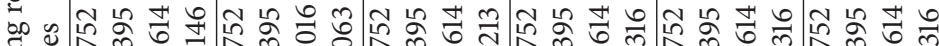

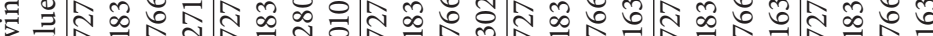

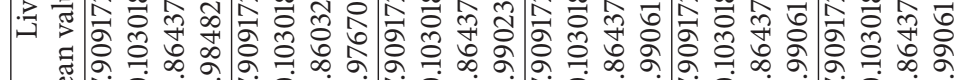

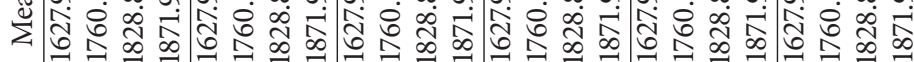

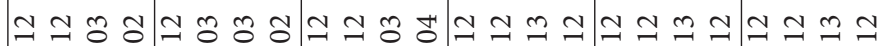
\begin{tabular}{lllllllllllllllllllllllll}
\hline & 1 & 1 & 1 & 1 & 1 & 1 & 1 & 1 & 1 & 1 & 1 & 1 & 1 & 1 & 1 & 1 & 1 & 1 & 1 & 1 & 1 & 1 & 1
\end{tabular}

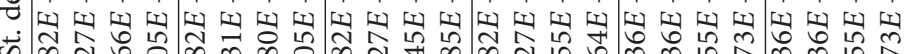

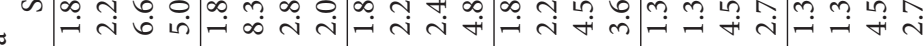

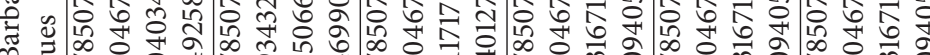

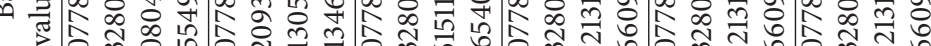
응 त్ర

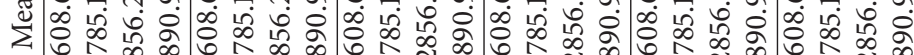
$\Sigma \mid \begin{gathered}\Delta \\ 0\end{gathered}$

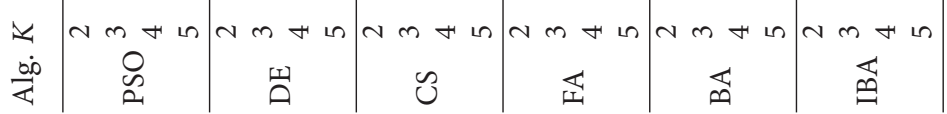




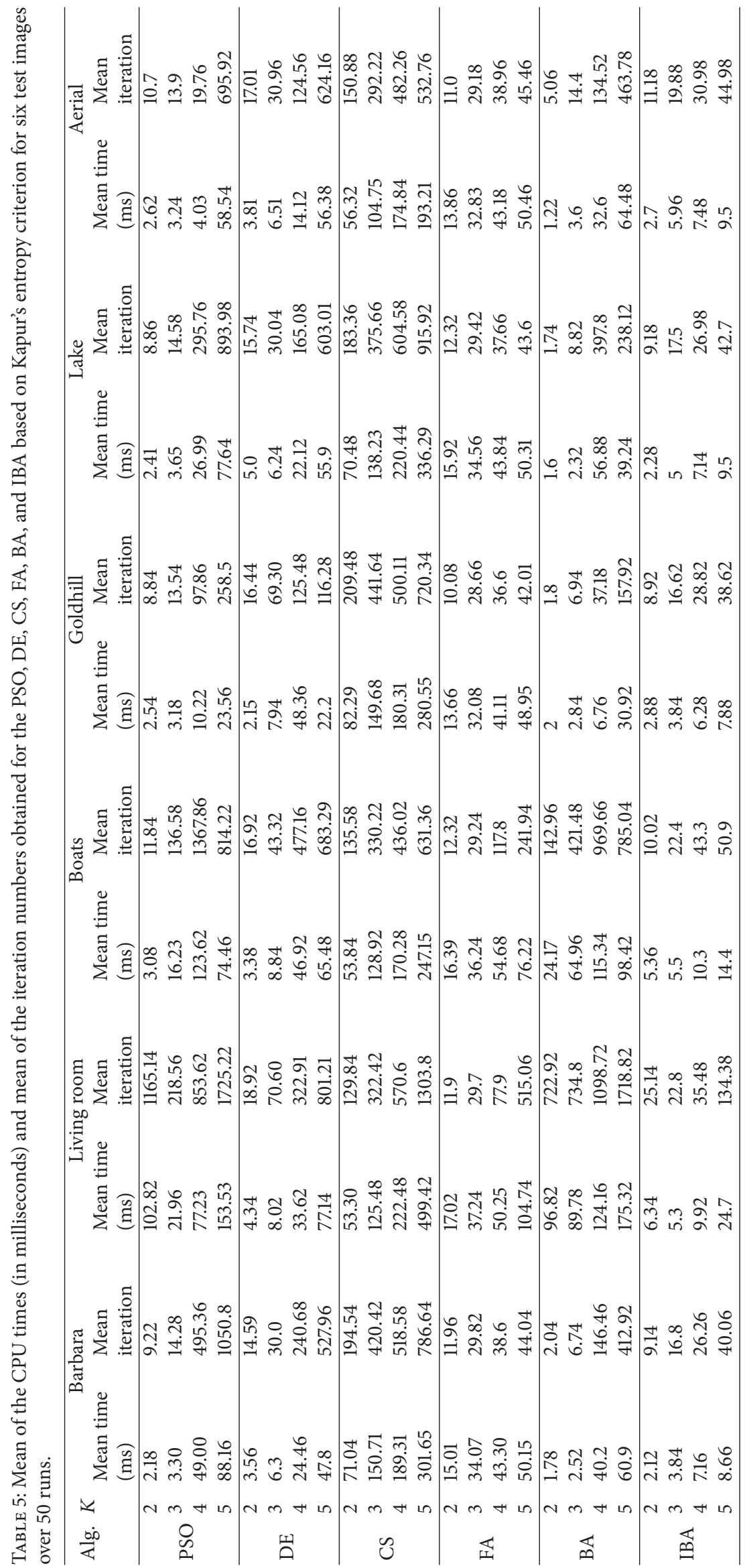




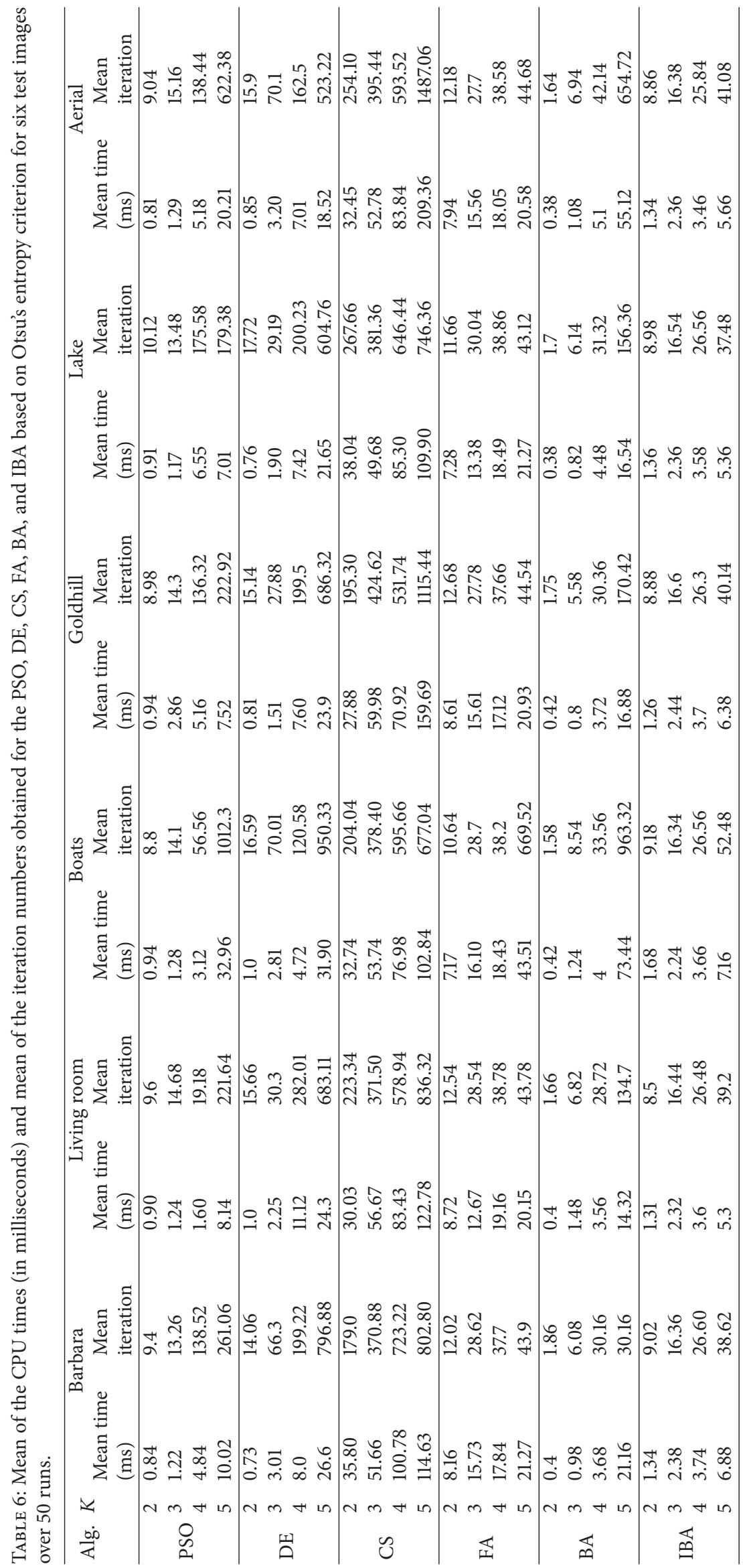


TABLE 7: The percent of the best results for thresholds 4 and 5 .

\begin{tabular}{lcc}
\hline Alg. & Kapur's method & Otsu's method \\
\hline PSO & $8 \%$ & $8 \%$ \\
DE & $0 \%$ & $0 \%$ \\
CS & $67 \%$ & $58 \%$ \\
FA & $67 \%$ & $92 \%$ \\
BA & $42 \%$ & $83 \%$ \\
IBA & $100 \%$ & $100 \%$ \\
\hline
\end{tabular}

TABLE 8: The number of evaluations for all test images and all threshold values for Kapur's method.

\begin{tabular}{lccccc}
\hline Alg. & Trsh. 2 & Trsh. 3 & Trsh. 4 & Trsh. 5 & Total \\
\hline PSO & 1214 & 411 & 3130 & 5439 & 10194 \\
DE & 96 & 186 & 1456 & 3356 & 5094 \\
CS & 1004 & 2183 & 3112 & 4891 & 11189 \\
FA & 70 & 176 & 347 & 932 & 1525 \\
BA & 876 & 1193 & 2784 & 3777 & 8631 \\
IBA & 74 & 116 & 192 & 352 & 734 \\
\hline
\end{tabular}

TABLE 9: The number of evaluations for all test images and all threshold values for Otsu's method.

\begin{tabular}{lccccc}
\hline Alg. & Trsh. 2 & Trsh. 3 & Trsh. 4 & Trsh. 5 & Total \\
\hline PSO & 56 & 85 & 665 & 25206 & 3326 \\
DE & 95 & 294 & 1164 & 4245 & 5798 \\
CS & 1323 & 2322 & 3669 & 5665 & 12979 \\
FA & 72 & 171 & 230 & 889 & 1362 \\
BA & 10 & 40 & 196 & 2110 & 2356 \\
IBA & 53 & 99 & 158 & 249 & 559 \\
\hline
\end{tabular}

method, the number of iterations does not grow rapidly with the increase of the number of thresholds as is the case with the rest of algorithms. From Table 8 we can also observe that the proposed IBA converges in considerably less iterations compared to the rest of algorithms.

From Table 9 (for the Otsu's criterion), it can be seen that the proposed IBA method in this case also converges in considerably less iterations compared to the other methods. It also maintains the feature of linearity with increasing the number of thresholds. Actually, in both cases, for Kapur's and Otsu's criteria, our proposed IBA algorithm improved the convergence speed by more than a factor of 2 , compared to the next best algorithm.

\section{Conclusion}

In this paper, we considered an important optimization problem of multilevel image thresholding. It is an exponential problem and as such it is appropriate for swarm intelligence metaheuristics. We adapted new bat algorithm for this problem and compared it to other state-of-the-art algorithms from [49]. Pure version of the bat algorithm performed well, but the results were slightly below the average, especially when Kapur's criterion was used. We determined that the pure bat algorithm, when applied to this problem, may be easily trapped into local optimum so we modified it by changing new solution equation by hybridized one with elements from DE. We also included limit parameter similar to the one used in the $\mathrm{ABC}$ algorithm.

Our proposed improved bat-inspired hybridized with DE (IBA) algorithm was tested on 6 standard benchmark images, the same as used in [49]. It proved to be superior to all other tested algorithms considering the quality of the solutions (it actually achieved the best result for both mean value and variance, for all tested cases), especially it significantly improved convergence speed (more than two times better than the next algorithm). This shows that our proposed algorithm is excellent choice for the multilevel image thresholding problem. Additional adjustments can be done in the future using larger set of synthetic images which will allow more precise modifications and parameter adjustment.

\section{Conflict of Interests}

The authors declare that there is no conflict of interests regarding the publication of this paper.

\section{Acknowledgment}

The research is supported by the Ministry of Science of Republic of Serbia, Grant no. III-44006.

\section{References}

[1] J. Lázaro, J. L. Martín, J. Arias, A. Astarloa, and C. Cuadrado, "Neuro semantic thresholding using OCR software for high precision OCR applications," Image and Vision Computing, vol. 28, no. 4, pp. 571-578, 2010.

[2] Y.-T. Hsiao, C.-L. Chuang, Y.-L. Lu, and J.A. Jiang, "Robust multiple objects tracking using image segmentation and trajectory estimation scheme in video frames," Image and Vision Computing, vol. 24, no. 10, pp. 1123-1136, 2006.

[3] R. Adollah, M. Y. Mashor, H. Rosline, and N. H. Harun, "Multilevel thresholding as a simple segmentation technique in acute leukemia images," Journal of Medical Imaging and Health Informatics, vol. 2, no. 3, pp. 285-288, 2012.

[4] A. Rojas Domínguez and A. K. Nandi, "Detection of masses in mammograms via statistically based enhancement, multilevelthresholding segmentation, and region selection," Computerized Medical Imaging and Graphics, vol. 32, no. 4, pp. 304-315, 2008.

[5] G. C. Anagnostopoulos, "SVM-based target recognition from synthetic aperture radar images using target region outline descriptors," Nonlinear Analysis: Theory, Methods and Applications, vol. 71, no. 12, pp. e2934-e2939, 2009.

[6] N. R. Pal and S. K. Pal, "A review on image segmentation techniques," Pattern Recognition, vol. 26, no. 9, pp. 1277-1294, 1993.

[7] N. Otsu, "A threshold selection method for grey level histograms," IEEE Transactions on Systems, Man and Cybernetics, vol. 9, no. 1, pp. 62-66, 1979.

[8] T. Pun, "A new method for grey-level picture thresholding using the entropy of the histogram," Signal Processing, vol. 2, no. 3, pp. 223-237, 1980. 
[9] T. Chaira and A. K. Ray, "Threshold selection using fuzzy set theory," Pattern Recognition Letters, vol. 25, no. 8, pp. 865-874, 2004.

[10] T. Chaira and A. K. Ray, "Segmentation using fuzzy divergence," Pattern Recognition Letters, vol. 24, no. 12, pp. 1837-1844, 2003.

[11] S. Wang, F. L. Chung, and F. Xiong, "A novel image thresholding method based on Parzen window estimate," Pattern Recognition, vol. 41, no. 1, pp. 117-129, 2008.

[12] A. Nakib, H. Oulhadj, and P. Siarry, "Non-supervised image segmentation based on multiobjective optimization," Pattern Recognition Letters, vol. 29, no. 2, pp. 161-172, 2008.

[13] S. S. Fan and Y. Lin, "A multi-level thresholding approach using a hybrid optimal estimation algorithm," Pattern Recognition Letters, vol. 28, no. 5, pp. 662-669, 2007.

[14] E. Zahara, S. S. Fan, and D.-M. Tsai, "Optimal multithresholding using a hybrid optimization approach," Pattern Recognition Letters, vol. 26, no. 8, pp. 1082-1095, 2005.

[15] M. Horng, "A multilevel image thresholding using the honey bee mating optimization," Applied Mathematics and Computation, vol. 215, no. 9, pp. 3302-3310, 2010.

[16] J. N. Kapur, P. K. Sahoo, and A. K. C. Wong, "A new method for gray-level picture thresholding using the entropy of the histogram," Computer Vision, Graphics, and Image Processing, vol. 29, no. 3, pp. 273-285, 1985.

[17] S. Zarezadeh and M. Asadi, "Results on residual Rényi entropy of order statistics and record values," Information Sciences, vol. 180, no. 21, pp. 4195-4206, 2010.

[18] P. K. Sahoo, S. Soltani, and A. K. C. Wong, "A survey of thresholding techniques," Computer Vision, Graphics and Image Processing, vol. 41, no. 2, pp. 233-260, 1988.

[19] X.-S. Yang, "Efficiency analysis of swarm intelligence and randomization techniques," Journal of Computational and Theoretical Nanoscience, vol. 9, no. 2, pp. 189-198, 2012.

[20] X.-S. Yang, "Review of meta-heuristics and generalised evolutionary walk algorithm," International Journal of Bio-Inspired Computation, vol. 3, no. 2, pp. 77-84, 2011.

[21] X.-S. Yang, "Free lunch or no free lunch: that is not just a question?" International Journal on Artificial Intelligence Tools, vol. 21, no. 3, Article ID 1240010, pp. 5360-5366, 2012.

[22] A. H. Gandomi and X.-S. Yang, "Evolutionary boundary constraint handling scheme," Neural Computing \& Applications, vol. 21, no. 6, pp. 1449-1462, 2012.

[23] J. Kennedy and R. Eberhart, "Particle swarm optimization," in Proceedings of the IEEE International Conference on Neural Networks (ICNN '95), vol. 4, pp. 1942-1948, December 1995.

[24] R. Storn and K. Price, "Differential evolution-a simple and efficient heuristic for global optimization over continuous spaces," Journal of Global Optimization, vol. 11, no. 4, pp. 341359, 1997.

[25] X.-S. Yang, "Firefly algorithms for multimodal optimization," in Stochastic Algorithms: Foundations and Applications, vol. 5792 of Lecture Notes in Computer Science, pp. 169-178, Springer, Berlin, Germany, 2009.

[26] I. Fister, I. Fister Jr., X.-S. Yang, and J. Brest, "A comprehensive review of firefly algorithms," Swarm and Evolutionary Computation, vol. 13, no. 1, pp. 34-46, 2013.

[27] X.-S. Yang and S. Deb, "Cuckoo search via Lévy flights," in Proceedings of the World Congress on Nature and Biologically Inspired Computing (NABIC '09), pp. 210-214, Coimbatore, India, December 2009.
[28] X.-S. Yang and S. Deb, "Engineering optimisation by cuckoo search," International Journal of Mathematical Modelling and Numerical Optimisation, vol. 1, no. 4, pp. 330-343, 2010.

[29] A. H. Gandomi, X.-S. Yang, and A. H. Alavi, "Cuckoo search algorithm: a metaheuristic approach to solve structural optimization problems," Engineering with Computers, vol. 29, no. 1, pp. 17-35, 2013.

[30] M. Dorigo and L. M. Gambardella, "Ant colonies for the travelling salesman problem," BioSystems, vol. 43, no. 2, pp. 7381, 1997.

[31] M. Tuba and R. Jovanovic, "Improved ACO algorithm with pheromone correction strategy for the traveling salesman problem," International Journal of Computers, Communications \& Control, vol. 8, no. 3, pp. 477-485, 2013.

[32] R. Jovanovic and M. Tuba, "An ant colony optimization algorithm with improved pheromone correction strategy for the minimum weight vertex cover problem," Applied Soft Computing Journal, vol. 11, no. 8, pp. 5360-5366, 2011.

[33] R. Jovanovic and M. Tuba, "Ant colony optimization algorithm with pheromone correction strategy for the minimum connected dominating set problem," Computer Science and Information Systems, vol. 10, no. 1, pp. 133-149, 2013.

[34] D. Karaboga, "An idea based on honey bee swarm for numerical optimization," Tech. Rep. TR06, Erciyes University, Engineering Faculty, Computer Engineering Department, 2005.

[35] N. Bacanin and M. Tuba, "Artificial bee colony (ABC) algorithm for constrained optimization improved with genetic operators," Studies in Informatics and Control, vol. 21, no. 2, pp. 137-146, 2012.

[36] I. Brajevic and M. Tuba, "An upgraded artificial bee colony (ABC) algorithm for constrained optimization problems," Journal of Intelligent Manufacturing, vol. 24, no. 4, pp. 729-740, 2013.

[37] M. Subotic and M. Tuba, "Parallelized multiple swarm artificial bee colony algorithm (MS-ABC) for global optimization," Studies in Informatics and Control, vol. 23, no. 1, pp. 117-126, 2014.

[38] M. Tuba and N. Bacanin, "Artificial bee colony algorithm hybridized with firefly metaheuristic for cardinality constrained mean-variance portfolio problem," Applied Mathematics \& Information Sciences, vol. 8, no. 6, pp. 2831-2844, 2014.

[39] X.-S. Yang, "A new metaheuristic bat-inspired Algorithm," Studies in Computational Intelligence, vol. 284, pp. 65-74, 2010.

[40] A. Alihodzic and M. Tuba, "Improved hybridized bat algorithm for global numerical optimization," in Proceedings of the 16th IEEE International Conference on Computer Modelling and Simulation (UKSim-AMSS '14), pp. 57-62, March 2014.

[41] C. Dai, W. Chen, Y. Song, and Y. Zhu, "Seeker optimization algorithm: A novel stochastic search algorithm for global numerical optimization," Journal of Systems Engineering and Electronics, vol. 21, no. 2, pp. 300-311, 2010.

[42] M. Tuba, I. Brajevic, and R. Jovanovic, "Hybrid seeker optimization algorithm for global optimization," Applied Mathematics \& Information Sciences, vol. 7, no. 3, pp. 867-875, 2013.

[43] M. Tuba and N. Bacanin, "Improved seeker optimization algorithm hybridized with firefly algorithm for constrained optimization problems," Neurocomputing, 2014.

[44] S. Sarkar, G. R. Patra, and S. Das, "A differential evolution based approach for multilevel image segmentation using minimum cross entropy thresholding," in Swarm, Evolutionary, and Memetic Computing, vol. 7076 of Lecture Notes in Computer Science, pp. 51-58, 2011. 
[45] P. Yin, "Multilevel minimum cross entropy threshold selection based on particle swarm optimization," Applied Mathematics and Computation, vol. 184, no. 2, pp. 503-513, 2007.

[46] B. Akay, "A study on particle swarm optimization and artificial bee colony algorithms for multilevel thresholding," Applied Soft Computing Journal, vol. 13, no. 6, pp. 3066-3091, 2013.

[47] M. Maitra and A. Chatterjee, "A hybrid cooperativecomprehensive learning based PSO algorithm for image segmentation using multilevel thresholding," Expert Systems with Applications, vol. 34, no. 2, pp. 1341-1350, 2008.

[48] K. Harnrnouche, M. Diaf, and P. Siarry, "A comparative study of various meta-heuristic techniques applied to the multilevel thresholding problem," Engineering Applications of Artificial Intelligence, vol. 23, no. 5, pp. 676-688, 2010.

[49] I. Brajevic and M. Tuba, "Cuckoo search and firefly algorithm applied to multilevel image thresholding," in Cuckoo Search and Firefly Algorithm: Theory and Applications, X.-S. Yang, Ed., vol. 516 of Studies in Computational Intelligence, pp. 115-139, Springer, Berlin, Germany, 2014.

[50] D. Campos, "Real and spurious contributions for the Shannon, Rényi and Tsallis entropies," Physica A, vol. 389, no. 18, pp. 37613768, 2010.

[51] M. Tuba, "Asymptotic behavior of the maximum entropy routing in computer networks," Entropy, vol. 15, no. 1, pp. 361371, 2013.

[52] G.-Q. Huang, W.-J. Zhao, and Q.-Q. Lu, "Bat algorithm with global convergence for solving large-scale optimization problem," Application Research of Computers, vol. 30, no. 5, pp. 13231328, 2013.

[53] X.-S. Yang and A. H. Gandomi, "Bat algorithm: A novel approach for global engineering optimization," Engineering Computations, vol. 29, no. 5, pp. 464-483, 2012.

[54] K. Khan, A. Nikov, and A. Sahai, "A fuzzy bat clustering method for ergonomic screening of office workplaces," in Advances in Intelligent and Soft Computing, vol. 101, pp. 59-66, Springer, 2011.

[55] L. Jiann-Horng, C. Chao-Wei, Y. Chorng-Horng, and T. HsienLeing, "A chaotic levy ight bat algorithm for parameter estimation in nonlinear dynamic biological systems," Journal of Computer and Information Technology, vol. 2, no. 2, pp. 56-63, 2012.

[56] X.-S. Yang, "Bat algorithm for multi-objective optimisation," International Journal of Bio-Inspired Computation, vol. 3, no. 5, pp. 267-274, 2011.

[57] J. Zhang and G. Wang, "Image matching using a bat algorithm with mutation," Applied Mechanics and Materials, vol. 203, no. 1, pp. 88-93, 2012.

[58] B. Ramesh, C. Jagan Mohan, and V. Reddy, "Application of bat algorithm for combined economic load and emission dispatch," International Journal of Electrical and Electronics Engineering \& Telecommunications, vol. 2, no. 1, pp. 1-9, 2013.

[59] F. A. Banu and C. Chandrasekar, "An optimized approach of modified BAT algorithm to record deduplication," International Journal of Computer Applications, vol. 62, no. 1, pp. 10-15, 2012.

[60] M. K. Marichelvam and T. Prabaharam, "A bat algorithm for realistic hybrid flowshop scheduling problems to minimize makespan and mean flow time," ICTACT International Journal on Soft Computing, vol. 3, no. 1, pp. 428-433, 2012.

[61] K. Khan and S. Ashok, "A comparison of ba, ga, pso, bp and $\mathrm{lm}$ for training feed forward neural networks in e-learning context," International Journal of Intelligent Systems and Applications, vol. 4, no. 7, pp. 23-29, 20112.
[62] R. Damodaram and M. L. Va larmathi, "Phishing website detection and optimization using modified bat algorithm," International Journal of Engineering Research and Applications, vol. 2, no. 1, pp. 870-876, 2012. 

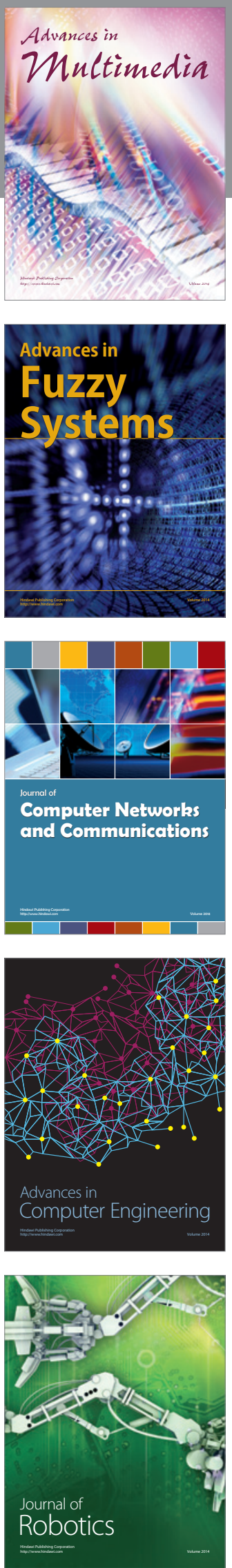

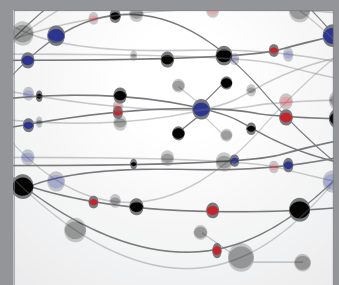

The Scientific World Journal
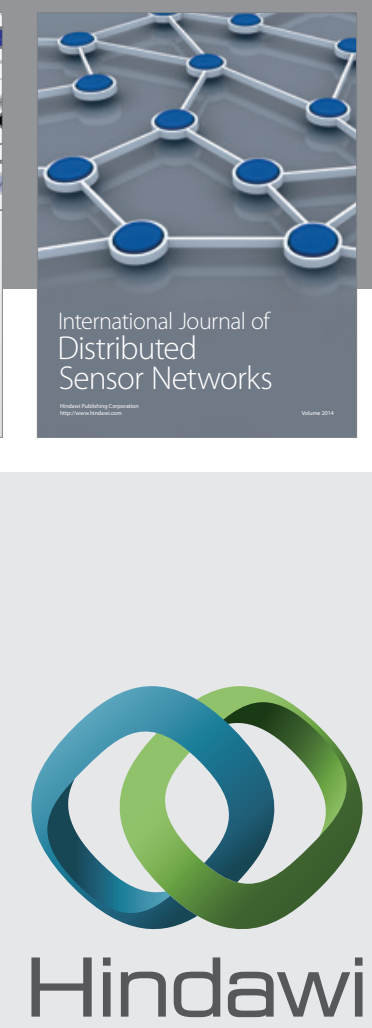

Submit your manuscripts at

http://www.hindawi.com
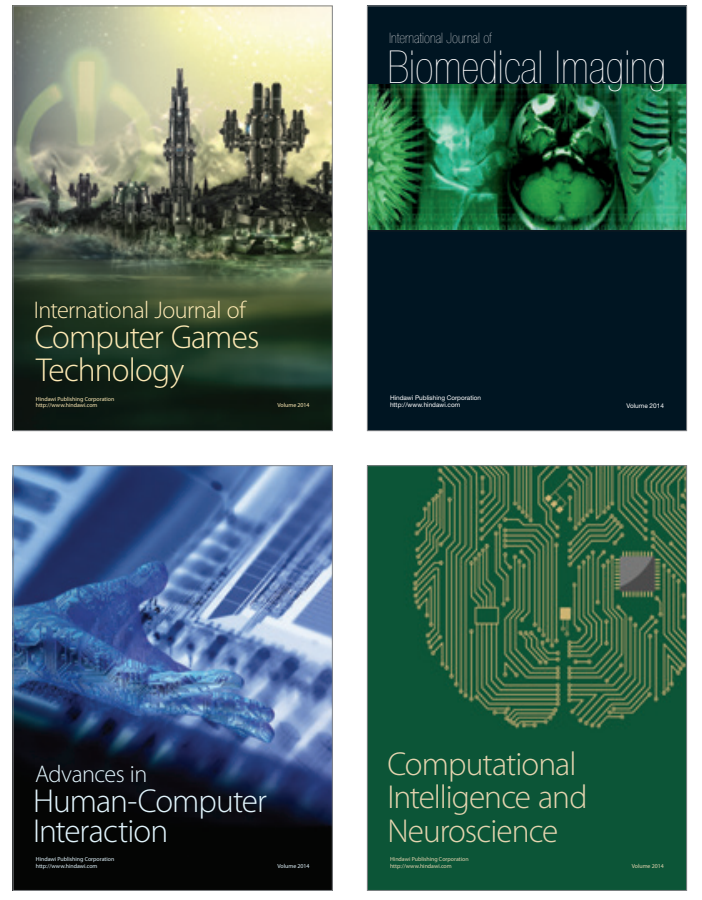
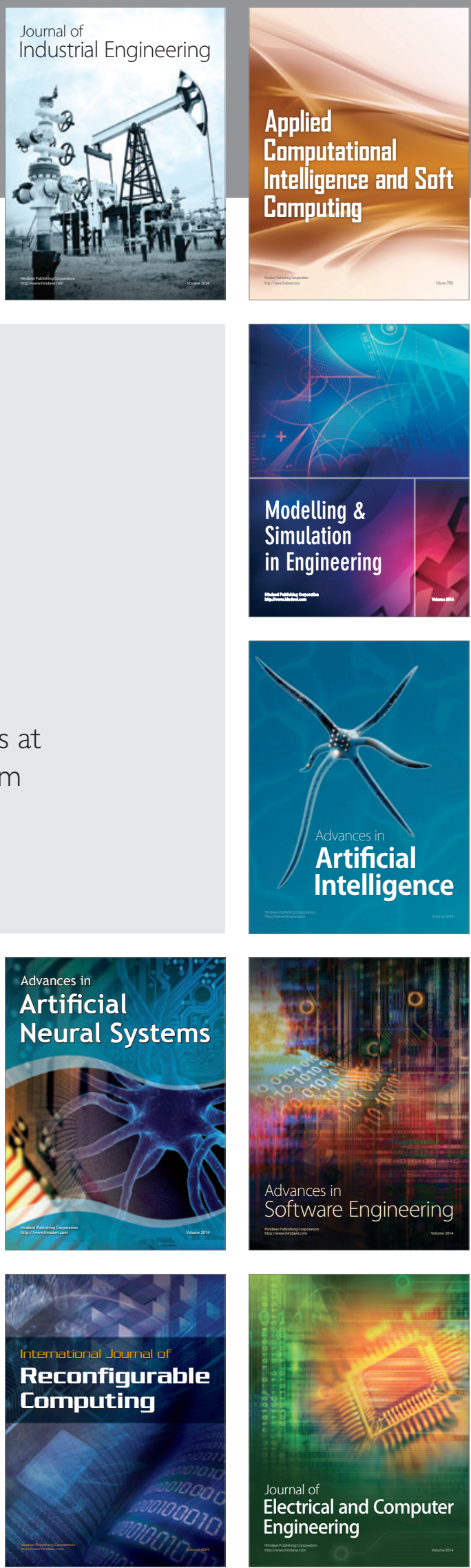